\title{
Tumour antigen-loaded mouse dendritic cells maturing in the presence of inflammatory cytokines are potent activators of immune response in vitro but not in vivo
}

\author{
JOANNA ROSSOWSKA, ELZBIETA PAJTASZ-PIASECKA, ANNA SZYDA, \\ AGNIESZKA KRAWCZENKO, NATALIA ZIETARA and DANUTA DUS
}

Ludwik Hirszfeld Institute of Immunology and Experimental Therapy, Polish Academy of Sciences, Wroclaw, Poland

Received October 20, 2008; Accepted January 28, 2009

DOI: 10.3892/or_00000386

\begin{abstract}
The use of dendritic cells (DCs) loaded with tumour antigen is one of the most promising approaches to induce tumour-specific immune response. However, methods of the vaccine preparation have not yet been standardized. The purpose of the study was to analyse the anti-tumour efficacy of tumour antigen-loaded mouse bone marrow-derived dendritic cells (BM-DC/TAg) at different maturation stages. BM-DCs were loaded with MC38 colon carcinoma cell lysate (TAg) alone, to become partially differentiated, or were additionally stimulated with inflammatory cytokines such as TNF- $\alpha$, IFN- $\gamma$, or IL-12 to reach complete maturity. BM-DCs simultaneously stimulated with TAg and cytokines (especially IL-12 or IFN- $\gamma+$ IL-12) were in vitro more effective immune response activators than BM-DC/TAg cells. However, the highest anti-tumour effect in vivo was noted when mice were treated just with BM-DC/TAg. In a further study, the ability of IL-12 gene transduced BM-DCs (BM-DC/IL-12) to augment the immune response induced by BM-DC/TAg cells at different stages of maturation was examined. The highest antitumour effect was observed when partially differentiated BM-DC/TAg cells were injected simultaneously with BM-DC/ IL-12 cells. The results suggest that partially differentiated BM-DC/TAg cells are more potent in evoking a strong antitumour response in vivo than mature BM-DCs. Moreover, the capacity of BM-DC/TAg cells for further differentiation and their sensitivity to factors secreted in vivo by the host or cells engineered to cytokine production seem to be of great importance.
\end{abstract}

Correspondence to: Dr Elzbieta Pajtasz-Piasecka, Institute of Immunology and Experimental Therapy, Polish Academy of Sciences, Laboratory of Experimental Anticancer Therapy, ul. R. Weigla 12, 53-114 Wroclaw, Poland

E-mail: pajtasz@iitd.pan.wroc.pl

Key words: murine colon carcinoma MC38, tumour antigen-loaded dendritic cells, inflammatory cytokines, $\mathrm{T}$ cell cytotoxicity, IFN- $\gamma$ production, CD107a molecules, IL-12 transduced BM-DC

\section{Introduction}

Dendritic cells (DCs) are the most effective among antigenpresenting cells. They can elicit both a primary immune response and boost a secondary immune response to foreign antigens $(1,2)$. Because of these properties, increasing attention has been directed to the use of DCs in vaccine strategies for cancer treatment. However, methods of vaccine preparation have not yet been standardized. It is still unclear which factors (e.g. inflammatory cytokines, antigenic stimulation) affect DC maturation and at which stage of differentiation DCs should be administered to recipients. It was reported that DCs can be loaded with various forms of tumour-associated antigens (TAA), including lysate of whole cells $(3,4)$, peptides (5), proteins (6), as well as RNA (7) or DNA (8). Among them, the use of whole tumour cell lysate became the most frequently described procedure. TAA can be processed by DCs and then presented by means of major histocompatibility complex (MHC) class I and class II molecules. The TAAMHC complexes trigger polyclonal expansion of both $\mathrm{CD}^{+}$ and CD8 ${ }^{+}$T cells (9-11). The consequence of DC loading with tumour-cell antigens is the limited ability of a tumour to escape the host's immune response. Nevertheless, it has been recently shown that tumour antigen-loaded DCs do not reach a completely mature stage and produce an amount of IL-12 insufficient to develop a strong anti-tumour response (12).

It is well known that pro-inflammatory cytokines such as $\mathrm{TNF} \alpha, \mathrm{IL}-1 \beta, \mathrm{IFN}-\gamma, \mathrm{IL}-12$, and prostaglandin E2 as well as non-inflammatory and pathogen-unrelated factors such as histamine, heparin, and ATP can modulate the DC maturation process in vivo (12-14). For example, TNF- $\alpha$ is a well-known factor able to induce high expression levels of MHC class II and co-stimulatory molecules on DCs, but is a weak stimulator of IL-12 production (15). IFN- $\gamma$ has a unique capacity to prime DCs for high IL-12 production, which could be accompanied by strong Th1-promotion (13). Interleukin 12 exhibits a number of important biological activities and can, among others, enhance the stimulatory capacity of bone marrowderived DCs in vitro (16). It has been demonstrated that these auxiliary stimuli diversify the capacities of DCs to prime appropriate $\mathrm{T}$ cell effector subsets (13) and to improve the $\mathrm{T}$ cell-mediated response in vitro (12).

On the basis of these findings we decided to determine whether tumour antigen-loaded bone marrow-derived dendritic 
cells (BM-DC/TAg) matured in the presence of inflammatory cytokines show the same strong activity in vivo as demonstrated in experiments performed in vitro. BM-DCs were loaded with MC38 colon carcinoma cell lysate (TAg) in the presence of TNF- $\alpha$, IFN- $\gamma$, or IL-12 (BM-DC/TAg+/cytokines) and then injected peritumourally into MC38 tumour-bearing mice as an anti-tumour vaccine. To assess the effect of such injections on development of cell-mediated immune response the ability of restymulated splenocytes to produce of IFN- $\gamma$ (Th1-associated cytokine) and IL-4 (Th2associated cytokine) was estimated. According to evidence that, mechanism of host cell cytotoxicity may be perforin/ granzyme-dependent the CD107a molecule was further investigated. CD107a (also known as lysosomal-associated membrane protein-1) is a vesicle membrane protein that becomes transiently mobilized to the cell surface during the degranulation process, while cytotoxic mediators such as perforin and granzymes are released (17-19). Thus, the expression of CD107a on surface of cytotoxic cells $\left(C D 8^{+} \mathrm{T}\right.$ cells and $\mathrm{CD} 49 \mathrm{~b}^{+} \mathrm{NK}$ cells) was estimated.

The presence of cytokines in the tumour microenvironment is considered as an important factor of host anti-tumour immunity induction. To restore the proper cytokine balance, cells engineered to produce cytokines have been increasingly applied in various tumour models. For example, intratumoural injection of DCs secreting IL-12 (DC/IL-12) was found to promote activation of IFN- $\gamma$ producing $\mathrm{CD} 4^{+}$and $\mathrm{CD} 8^{+} \mathrm{T}$ cells (20). These results suggested that a combination of DC/IL-12 and tumour antigen-loaded DCs (especially those matured in the presence of inflammatory cytokines) may enhance multiple DC effector functions and, consequently, accelerate immunemediated tumour rejection. Taking that into consideration, in the presented study we examined the efficacy of a vaccine consisting of BM-DCs transduced with pSAMEN vector carrying the mouse IL-12 gene (BM-DC/IL-12) and BM-DC/ TAg cells at different maturation stages.

\section{Materials and methods}

Mice. Experiments were performed using 6- to 8-week-old female C57BL/6 mice, which were finally sacrificed according to properly established protocols and government regulations (permission of the Local Ethics Committee, 2003).

Cell lines and culture media. RPMI-1640 culture medium (Gibco) was supplemented with $100 \mathrm{U} / \mathrm{ml}$ penicillin, $100 \mathrm{U} / \mathrm{ml}$ streptomycin, $2 \mathrm{mM}$ L-glutamine, $1 \mathrm{mM}$ sodium pyruvate and $50 \mu \mathrm{M}$ 2-mercaptoethanol (complete medium, CM). Bone marrow-derived dendritic cells (BM-DCs) were cultured in CM supplemented with $10 \%$ heat-inactivated fetal bovine serum (FBS, HyClone). An established mouse colon carcinoma cell line (MC38/0) (21) was cultured in CM supplemented with $5 \%$ FBS.

Generation of bone marrow-derived DCs (BM-DCs). Briefly, the long bones of the female C57BL6 mice were dislodged and the remaining tissue and muscles were removed with sterile gauze. The ends of the bones were cut with sterile scissors and the bone marrow was flushed from the femurs and tibias with $5 \% \mathrm{CM}$. The bone marrow was washed three times and the cells were re-suspended in CM supplemented with $10 \%$ (v/v) FBS $(10 \% \mathrm{CM})$. The cells were cultured in $75-\mathrm{cm}^{2}$ flasks at a concentration of $8 \times 10^{6}$ cells per flask in $10 \mathrm{ml}$ of $10 \% \mathrm{CM}$ supplemented with $40 \mathrm{ng} / \mathrm{ml}$ recombinant murine GM-CSF (Strathman) and IL-4. The source of murine IL-4 was X63mIL4 plasmacytoma cell supernatant used at a concentration of $1-5 \%$, corresponding to $100 \mathrm{U} / \mathrm{ml}$. The CM was replaced every second day. After six days the BM-DCs were used for further tests.

BM-DC stimulation with tumour antigen. MC38/0 cell lysate (named here tumour antigen: TAg) was prepared by five cycles of freezing in liquid nitrogen and thawing at $37^{\circ} \mathrm{C}$. Then the mixture was sonificated for $2 \mathrm{~h}$. The lysate was frozen and stored at $-20^{\circ} \mathrm{C}$. Immature BM-DCs were seeded in $75-\mathrm{cm}^{2}$ flasks and then loaded with TAg for $24 \mathrm{~h}$ (TAg was added to the BM-DCs at a proportion corresponding to a BM-DC/ tumour-cell ratio of $1: 1)$. Maturation-inducing cytokines (100 U/ml IFN- $\gamma, 500 \mathrm{U} / \mathrm{ml} \mathrm{TNF-} \alpha$ and $50 \mathrm{ng} / \mathrm{ml} \mathrm{IL-12,} \mathrm{all}$ purchased from R\&D Systems) were added $2 \mathrm{~h}$ after TAg. The incubation was stopped and all stimulators were removed by centrifugation after $24 \mathrm{~h}$.

Retroviral transduction. The murine BM-DCs were transduced with the retroviral vector SAMEN IL-12 carrying murine IL-12 genes (mIL-12 p35 and p40) according to a procedure described previously (22). The cells were incubated for $24 \mathrm{~h}$ with virus containing supernatant supplemented with $40 \mathrm{ng} / \mathrm{ml}$ GM-CSF and $8 \mu \mathrm{g} / \mathrm{ml}$ polybrene. After transduction the cells were cultured in fresh medium for at least $48 \mathrm{~h}$. Temporarily IL-12-transduced BM-DCs (BM-DC/IL-12) were thus obtained. The cells, settled in a 24 -well TC plate with $0.5 \times 10^{6}$ cells $/$ well $/ \mathrm{ml}$ of $5 \% \mathrm{CM}$ for $48 \mathrm{~h}$, produced $\sim 2000 \mathrm{pg} / \mathrm{ml}$ of IL-12 as estimated by ELISA assay (BD Pharmingen).

Flow cytometric analysis of cell-surface phenotype. BM-DCs were collected for the phenotypical characteristics in cold PBS without $\mathrm{Ca}^{2+}$ and $\mathrm{Mg}^{2+}\left(\mathrm{PBS}^{-}\right)$containing $2.5 \% \mathrm{FBS}$, washed, and then incubated with the fluorophore-labeled monoclonal antibodies (mAbs) rat anti-mouse RPE-CD80 (Serotec, clone RMMP-1), rat anti-mouse RPE-CD86 (BD Pharmingen, clone GL1), rat anti-mouse PE-CD40 (BD Pharmingen, clon 3/23) and the appropriate RPE-labeled IgG isotype controls (Serotec, IgG2a, clone LO-DNP-16; BD Pharmingen, IgG1k, clone R3-34). In two-step test BM-DC were incubated with mouse anti-mouse $\mathrm{H}-2 \mathrm{~K}^{\mathrm{b}} \mathrm{D}^{\mathrm{b}}$ (BD Pharmingen, clone 28-8-6), mouse anti-mouse I-A ${ }^{\mathrm{b}}$ (BD Pharmingen, clone AF6-120.1) and second reagent FITC-labeled rat anti-mouse (BD Pharmingen, clone R-240). The cells were stained for $45 \mathrm{~min}$ at $4^{\circ} \mathrm{C}$ in the one-step test and 30 and $45 \mathrm{~min}$, respectively, in the two-step test. The analysis was carried out using a Becton-Dickinson FACSCalibur apparatus with Cell Quest Software.

Induction of Th1/Th2 response by BM-DCs. BM-DCs previously stimulated with TAg and different cytokine combinations as well as TAg-loaded BM-DCs alone were co-cultured with naïve splenocytes for five days (BM-DC/ splenocytes at a ratio of $1: 10)$. The percentage of IFN- $\gamma$ producing $\mathrm{CD}^{+}$cells after stimulation with BM-DCs was measured by intracellular cytokine staining test. During the 
last 5-6 $\mathrm{h}$ of the five-day incubation, the cells were treated with brefeldin A $\left(5-10 \mu \mathrm{g} / \mathrm{ml}\right.$ at $37^{\circ} \mathrm{C}$, Sigma). Then the cells were harvested, re-suspended in $\mathrm{PBS}^{-}$and centrifuged. The cells were fixed and permeabilized for $20 \mathrm{~min}$ at $4^{\circ} \mathrm{C}$ by Cytofix/Cytoperm (Pharmingen), then washed and suspended in PBS- $^{-}$at a density of $1 \times 10^{6}$ cells $/ \mathrm{ml}$. For intracellular cell staining, RPE-conjugated rat anti-mouse IFN- $\gamma$ mAb (BD Pharmingen, clone XMG1.2) and the appropriate Ig isotype control (clone R3-34) in a final dilution 1:400 were used. The $50-\mu 1$ cell aliquots were mixed with $50 \mu 1$ of antibodies and incubated at room temperature for $40 \mathrm{~min}$. The analysis was carried out using a Becton-Dickinson FACSCalibur apparatus with Cell Quest Software. Additionally, supernatants from a mixed culture of BM-DCs and splenocytes were collected and stored at $-20^{\circ} \mathrm{C}$ and then analysed using commercially available ELISA kits (BD Pharmingen) to measure IFN- $\gamma$ production.

Mouse-treatment schedule. Mice were inoculated subcutaneously (s.c.) with MC38/0 cells $\left(1 \times 10^{6}\right.$ in $0.2 \mathrm{ml} /$ mouse) Fourteen days after tumour inoculation, when the tumours were palpable, the animals were randomly divided into groups of five or six with similar tumour volumes. Mice were injected three times peritumourally (p.t.) with BM-DC/TAg and/or BM-DC/IL-12 cells (ca. 1x10\%/mouse, once a week). The control mice were given $0.2 \mathrm{ml}$ of physiological saline. The mice were monitored twice a week and the tumour volume was estimated by measuring the tumour size and using the formula $\mathrm{a} / 2 \mathrm{x} \mathrm{b}^{2}$, where a represents the largest and $\mathrm{b}$ the smallest tumour diameter. The time required for the tumour to reach a volume of $1 \mathrm{~cm}^{3}$ (TRV, days) was evaluated graphically from median tumour growth curves. Tumour growth inhibition (TGI, \%) was calculated according to the formula 100 - (MVT/MVC x100) (\%), where MTV is the median tumour volume in treated mice and MVC the median tumour volume in control mice. Statistical differences in tumour growth inhibition (measured for median tumour volume) were calculated after the third injection of every experiment, using the Kruskal-Wallis U-test. Differences with $\mathrm{p}<0.05$ were regarded as significant.

Estimation of cytotoxicity and IFN- $\gamma$ and IL-4 production. Splenocytes from healthy mice, untreated MC38 tumourbearing mice, mice on the seventh day after the third injection and from mice which survived up to one month after the third injection of BM-DC-based vaccines were used. Splenocytes were isolated and co-cultured (restimulated) for five days with mitomycin C-treated MC38 cells (50 $\mu \mathrm{g}$ mitomycin $\mathrm{C} / 3 \times 10^{6}$ cells $/ \mathrm{ml}$ of $5 \% \mathrm{CM}$ incubated for $30 \mathrm{~min}$ at $37^{\circ} \mathrm{C}$ ) in the presence of IL-2. The source of murine IL-2 was X63mIL-2 plasmacytoma cell supernantant used at a concentration of $3-5 \%$, corresponding to $100 \mathrm{U} / \mathrm{ml}$. After five days of restimulation, the culture supernatants were collected and stored at $-20^{\circ} \mathrm{C}$. To measure IFN- $\gamma$ and IL-4 production by activated splenocytes, commercially available ELISA kits (Pharmingen) were used.

The cytotoxicity of the splenocytes was tested by flow cytometry. Target MC38/0 cells were stained with $\mathrm{DiOC}_{18}$ lipophilic dye (Molecular Probes) for $30 \mathrm{~min}$ at $37^{\circ} \mathrm{C}$ and then washed with $2.5 \%$ PBS- ${ }^{-}$The spenocytes were incubated after restimulation with labeled target cells for $4 \mathrm{~h}$ at $37^{\circ} \mathrm{C}$ at a 10:1 ratio of effector to target cells. Then the cells were washed and dead cells were stained with propidium iodide (PI). Samples were analysed by flow cytometry on a FACS Calibur using the FL1 and FL3 filters. The percentage of dead cells (PI-positive) among the $\mathrm{DiOC}_{18}$-labeled target cells was calculated.

Detection of $\mathrm{CD}^{+} \mathrm{CD} 107 \mathrm{a}^{+}$and $\mathrm{CD}^{+} \mathrm{Cb}^{+} \mathrm{CD} 107 \mathrm{a}^{+}$cells among restimulated splenocytes. Restimulated splenocytes were co-cultured with $\mathrm{MC} 38 / 0$ target cells for $4 \mathrm{~h}$ at $37^{\circ} \mathrm{C}$ at a 10:1 effector to target cell ratio. Rat anti-mouse CD107a $\mathrm{mAb}$ (BD Pharmingen, clone 1D4B) was added to the cell suspension. After incubation with MC38/0 cells and $\mathrm{mAb}$ splenocytes were harvested, resuspended in $2.5 \% \mathrm{PBS}^{-}$, centrifuged, and then stained with AlexaFluor 488-conjugated goat anti-rat $\mathrm{mAb}$ (Molecular Probes) for $45 \mathrm{~min}$ at $4^{\circ} \mathrm{C}$. The excess of $\mathrm{mAb}$ was washed out and the splenocytes were stained with PE-conjugated rat anti-mouse CD8 mAb (Sigma, clone 53-6.7) or PE-conjugated rat anti-mouse CD49b mAb (BD Pharmingen, Pan-NK cells, clone DX5) for further $45 \mathrm{~min}$ at $4^{\circ} \mathrm{C}$ and then analysed using the Becton-Dickinson FACSCalibur apparatus with Cell Quest Software.

\section{Results}

TAg-loaded BM-DCs do not reach a completely mature stage. Immature mouse bone marrow-derived cells (BM-DCs), characterised by low expression of co-stimulatory and MHC class I and II molecules, were maintained in 10\% CM supplemented with GM-CSF and IL-4 for seven days as described above. The quality and purity of the BM-DCs were estimated by the percentage of CD $11 \mathrm{c}^{+}$cells, amounting to $\sim 85 \%$ and assessed before each assay performed. To obtain mature and tumour antigen-presenting BM-DCs, cells were loaded with mouse colon carcinoma cell lysate (TAg) for $24 \mathrm{~h}$ (BM-DC/TAg). However, there was no increase in levels of surface marker expression compared with unloaded BM-DCs (Fig. 1). Expression of CD80 and CD40 co-stimulatory molecules was similar for both groups, whereas the expression of such antigens as CD86, MHC I, and MHC II was slightly lower on BM-DCs/TAg cells compared with unloaded BMDCs. For this reason, we decided to apply additional maturation stimuli to reach optimal conditions for the maturation of BM-DCs. BM-DC cells were loaded with TAg for $2 \mathrm{~h}$ and for further $22 \mathrm{~h}$ stimulated with: IFN- $\gamma(\mathrm{BM}-\mathrm{DC} / \mathrm{TAg}+\mathrm{IFN}-\gamma)$, IL-12 (BM-DC/TAg+IL-12), TNF- $\alpha$ (BM-DC/TAg+TNF- $\alpha$ ), or combinations of IFN- $\gamma$ and TNF- $\alpha$ (BM-DC/TAg+IFN- $\gamma$ $+\mathrm{TNF}-\alpha)$ or IFN- $\gamma$ and IL-12 (BM-DC/TAg+IFN- $\gamma+$ IL-12). The presence of inflammatory cytokines in the BM-DC culture medium evoked increase in expression of DC maturation markers. The most significant differences were observed when IL-12, IFN- $\gamma+$ TNF- $\alpha$, or IFN- $\gamma+$ IL-12 were added (Fig. 1).

BM-DC/TAg cells maturing in the presence of inflammatory cytokines induce a $T$ cell response in vitro. BM-DC/TAg cells at different stages of maturation were co-cultured with naïve splenocytes for five days. Then the intracellular expression of IFN- $\gamma$ in $\mathrm{CD}^{+}$cells as well as the concentration of IFN- $\gamma$ in 5 day supernatants collected from the BM-DC and T cell 


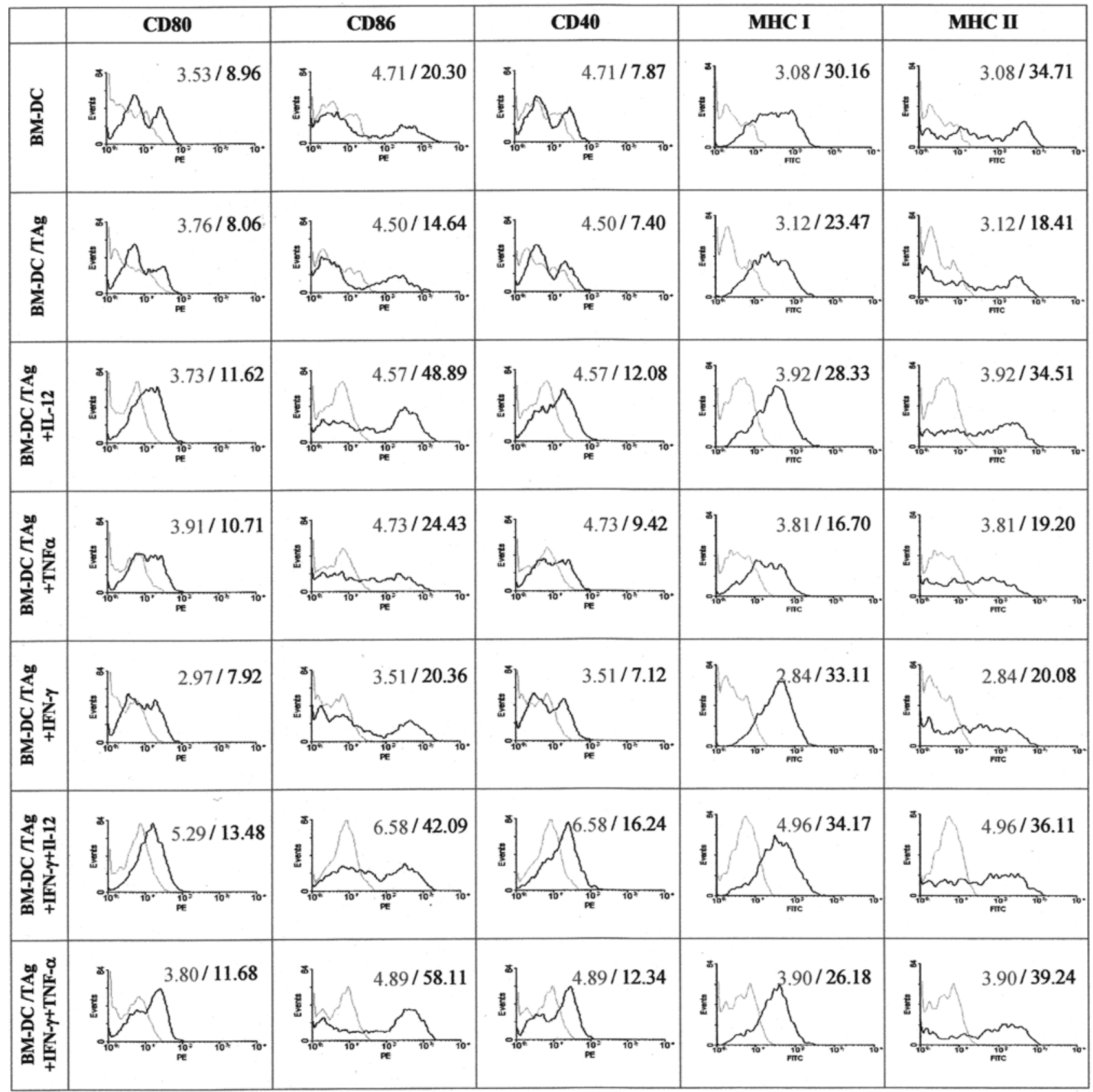

Figure 1. Phenotype characteristics of BM-DCs loaded with tumour antigen (TAg) and treated with inflammatory cytokines TNF- $\alpha$, IFN- $\gamma$, IL-12, or their combinations. The expression of CD80, CD86, CD40, and MHC I and MHC II was estimated after $24 \mathrm{~h}$ of incubation with TAg and the cytokines. The figure presents results from one of four experiments. The numbers correspond to MFI (mean florescence intensity) for the isotype control (gray) vs. the examined surface antigen (black).

co-culture were examined. The in vitro activation of $\mathrm{T}$ cells was dependent on the stage of BM-DC/TAg maturity. BM-DC/TAg stimulated with a combination of IFN- $\gamma$ and IL-12 appeared to be the best activators of cytotoxic T cells. In this case, as much as $34.6 \%$ of $\mathrm{CD}^{+}$cells from whole analysed population produced IFN- $\gamma$ (Fig. 2E), whereas only $3.4 \%$ did so after stimulation with partially differentiated BM-DC/TAg cells (stimulated only with TAg; Fig. 2A). The further comparisons of the percentages of IFN- $\gamma$-producing $\mathrm{CD} 8^{+}$cells generated by BM-DC/TAg+/- cytokines are shown in Fig. 2 . The analysis of IFN- $\gamma$ concentration in the collected supernatants demonstrated that depending on the type of stimulating BM-DCs, splenocytes were able to produce various amount of IFN- $\gamma$ : from $23 \mathrm{ng} / \mathrm{ml}$ after contact with BM-DC/ $\mathrm{TAg}+\mathrm{IFN}-\gamma+\mathrm{TNF}-\alpha$ cells up to $108 \mathrm{ng} / \mathrm{ml}$ if they were activated by matured BM-DC/TAg+IFN- $\gamma+$ IL-12 cells (Fig. 3 ).

The use of BM-DC/TAg cells at different stages of maturity for the treatment of mice with an established MC 38 tumour. To verify whether BM-DC/TAg cells maturing in the presence of exogenous cytokines could affect anti-tumour response in vivo more efficiently than BM-DC/TAg cells 


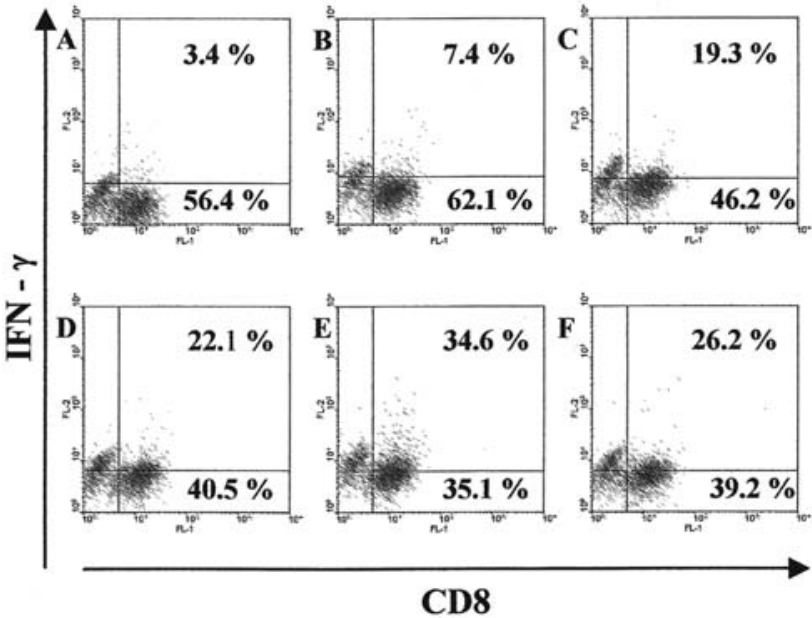

Figure 2. The percentage of IFN- $\gamma$-producing $\mathrm{CD} 8^{+}$cells after activation by BM-DCs. BM-DCs were treated with TAg and with cytokines for $24 \mathrm{~h}$ and then co-cultured with naive splenocytes at a BM-DC/T-cell ratio of 1:10 for 5 days. After brefeldin A treatment the cells were incubated with anti-CD8 anibody conjugated with FITC, then permeabilized and stained with antiIFN- $\gamma$ - PE antibody. Splenocytes were primed with (A) TAg-loaded BMDCs (BM-DC/TAg), (B) BM-DC/TAg+IL-12, (C) BM-DC/TAg+TNF- $\alpha$, (D) BM-DC/TAg+IFN- $\gamma$, (E) BM-DC/TAg+IFN- $\gamma+\mathrm{IL}-12$, (F) BM-DC TAg+IFN- $\gamma+\mathrm{TNF}-\alpha$. Actual percentages of $\mathrm{CD}^{+}$(lower right corner) and $\mathrm{CD} 8+\mathrm{IFN}-\gamma^{+}$(upper right corner) cells are shown from one representative experiment.

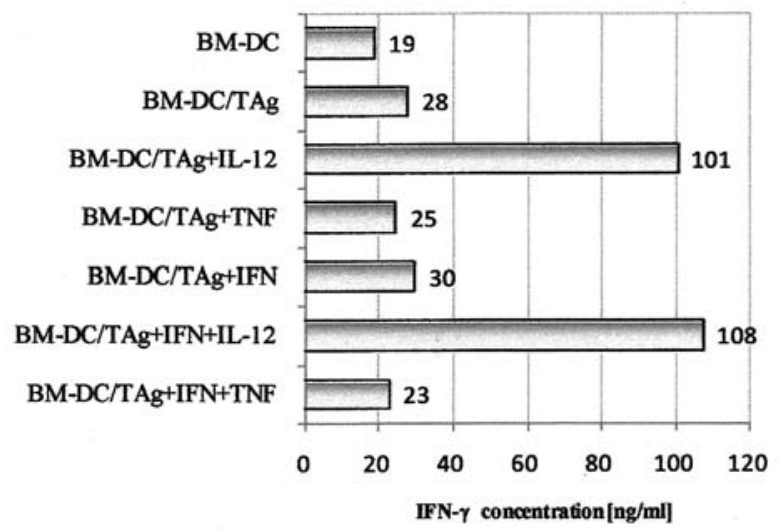

Figure 3 . IFN- $\gamma$ production by spleen cells primed by BM-DC/TAg maturing in the presence of inflammatory cytokines. The values represent the cytokine concentrations $(\mathrm{ng} / \mathrm{ml})$ in medium after five days of co-culture of BM-DC/TAg with spleen cells at a 1:10 ratio.

alone, C57BL/6 mice with an established MC38 tumour were injected peritumourally with BM-DC-based vaccines. The following cell groups were applied: BM-DC/TAg, BM-DC/ $\mathrm{TAg}+\mathrm{IFN}-\gamma, \mathrm{BM}-\mathrm{DC} / \mathrm{TAg}+\mathrm{TNF}-\alpha, \mathrm{BM}-\mathrm{DC} / \mathrm{TAg}+\mathrm{IL}-12$, BM-DC/ TAg+IFN- $\gamma+$ IL-12 or BM-DC/TAg+IFN- $\gamma+$ TNF- $\alpha$. Cells $\left(\sim 1 \times 10^{6}\right.$ cells per mouse) were injected once a week for three consecutive weeks. The injection of the BM-DC-based vaccines resulted only in insignificant tumour growth delay, which was presented as the time required for the tumour to acquire a volume of $1 \mathrm{~cm}^{3}$ (TRV). Difference in TRV ( $\left.\Delta T R V\right)$ reached 2-4 days compared with the control mice (Fig. 4). The percentages of tumour-growth inhibition (TGI), calculated on the $7^{\text {th }}$ day after the last injection, ranged from 29 to
$44 \%$ (Fig. 4). Despite the similar values of $\Delta \mathrm{TRV}$ and TGI for tested vaccines and no statistically significant differences in median tumour volumes in particular groups compared with control, the partially differentiated BM-DC/TAg cells seem to be the best activators of anti-tumour response ( $\triangle \mathrm{TRV}$ - 4 days and TGI $44 \%$ ).

To assess the impact of BM-DC/TAg+/- cytokine cells on host immune-cell activity, seven days (Fig. 5 cell groups A-G) and one month after the third injection of vaccine cells (Fig. 5 cell groups $\mathrm{H}-\mathrm{K}$ ) the animals were sacrificed and their spleens were dissected. The in vivo primed splenocytes were co-cultured for five days with MC38/0 cells (restimulation) and then their cytotoxic activity as well as their ability to produce IFN- $\gamma$ and IL-4 were estimated. An increase in cytotoxic activity of restimulated splenocytes was observed only one month after the third BM-DC injection. The highest cytotoxic activity was noted after BM-DC/TAg injection (40\%; Fig. 5I), although the differences in the action of the particular BM-DC vaccines were insignificant. BM-DC/TAg cells maturing in the presence of inflammatory cytokines proved to be less effective immune system activators and the cytotoxic activity of the splenocytes ranged from 32 to $37 \%$, whereas that of the untreated control mice reached $32 \%$. In contrast to the time-related increase in cytotoxic activity of splenocytes, their highest capacity to produce IFN- $\gamma$ was only noted seven days after the third BM-DC vaccination (Fig. 5II). Restimulated splenocytes from mice injected with BM-DC/ TAg+IFN $\gamma$, BM-DC/TAg+IL12, or BM-DC/TAg cells produced $\sim 9 \mathrm{ng} / \mathrm{ml}$ of IFN- $\gamma$, while those from mice injected with BM-DC/TAg+IFN- $\gamma+$ IL-12 or BM-DC/TAg+IFN- $\gamma$ $+\mathrm{TNF}-\alpha$ cells produced $\sim 7 \mathrm{ng} / \mathrm{ml}$. Splenocytes obtained one month after BM-DC administration did not produce IFN- $\gamma$ (Fig. 5II).

Since anti-tumour cytotoxicity was considered to be perforin/granzyme-dependent, we checked the expression of CD107a on the surface of the restimulated splenocytes. Among the splenocytes obtained on the seventh day after the third BM-DC injection there was a low number of CD107 $\mathrm{a}^{+}$ cells (from 6.2 to $8.3 \%$ for BM-DC/TAg+IFN- $\gamma+\mathrm{IL}-12$ and BM-DC/TAg+IFN- $\gamma+$ TNF- $\alpha$ cells respectively), whereas one month after BM-DC injection the percentage of $\mathrm{CD} 107 \mathrm{a}^{+}$cells ranged from $11.3 \%$ (BM-DC/TAg+IFN- $\gamma+\mathrm{TNF}-\alpha$ cells) to $17.5 \%$ (BM-DC/TAg+IFN $\gamma$ cells) (Fig.5III). CD107a molecules appeared mainly on the surface of $\mathrm{CD}^{+}$cells and the percentage of double-positive cells one month after the third BM-DC injection was about twice that of the sevenday time-period (Fig. 5III).

In the case of $\mathrm{CD}_{4} 9 \mathrm{~b}^{+}$cells, a similar phenomenon was noted. The percentage of CD107a+ CD $49 \mathrm{~b}^{+}$cells was much higher one month after than on day 7 after the third BM-DC injection (Fig. 5IV). Nevertheless, taking the different types of BM-DC-based vaccine into consideration, the differences in the number of $\mathrm{CD} 107 \mathrm{a}^{+} \mathrm{CD} 8^{+}$or $\mathrm{CD} 107 \mathrm{a}^{+} \mathrm{CD} 49 \mathrm{~b}^{+}$cells were not significant. Generally, the obtained data reflect a considerable tendency of gradual increase in percentage of cytolytic effector splenocytes and sudden decrease IFN- $\gamma$ production.

Do BM-DC/IL-12 cells applied together with fully matured $B M-D C / T A g$ induce a more effective immune response? In a 


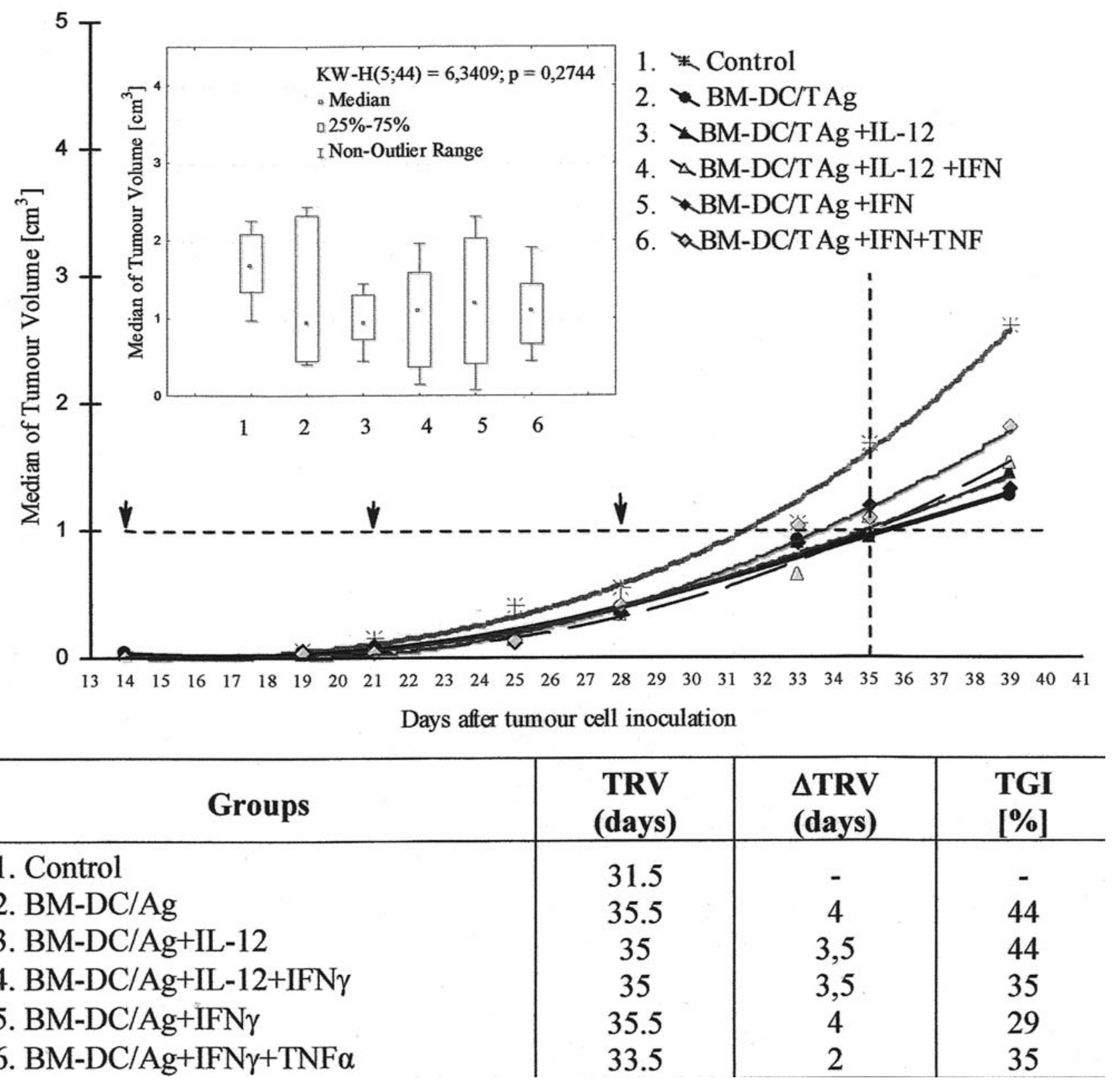

Figure 4. MC38 colon tumour growth in C57BL/6 mice treated with multiple peritumoural injections of BM-DC/TAg cells maturing in the presence of inflammatory cytokines. Mice bearing advanced s.c. growing MC38 tumours (7-8 per group until day 35 of observation) were injected p.t. $\left(\sim 0.7-0.9 \times 10^{6}\right.$ cells per injection) on three consecutive weeks (arrows in the graph). TRV, time in days required for tumour to reach a volume of $1 \mathrm{~cm}^{3} ; \Delta \mathrm{TRV}$, difference between the TRVs of treated mice and control mice; TGI, tumour growth inhibition calculated on day 35 after tumour inoculation (one week after the third BM-DC injection). Differences at $\mathrm{p}<0.05$ were regarded as significant.

further investigation we checked whether BM-DCs transduced with IL-12 gene (BM-DC/IL-12) were able to enhance the anti-tumour effect of BM-DC/TAg cells matured in the presence of inflammatory cytokines. The BM-DC/IL-12 cells possessed features of partially differentiated BM-DCs. They showed increased expressions of co-stimulatory molecules, especially CD86 and CD40 as well as class II MHC antigens, compared with unmodified BM-DCs (Fig. 6). BM-DC/IL-12 cells were also able to activate naïve splenocytes in vitro to IFN $-\gamma$ production. Moreover, simultaneous application of BM-DC/IL-12 with partially differentiated BM-DC/TAg cells evoked enhanced $\mathrm{T}$ cell activation in vitro and consequently increased IFN- $\gamma$ production than BM-DC/IL-12 with BM-DC/TAg+cytokines (data not shown).

The anti-tumour activity of BM-DC/TAg, BM-DC/ $\mathrm{TAg}+\mathrm{IFN}-\gamma$ and BM-DC/TAg+IFN- $\gamma+\mathrm{TNF}-\alpha$ cells and their combinations with BM-DC/IL-12 cells was further estimated in vivo. In this experiment, MC38 tumour-bearing mice were vaccinated once a week for three consecutive weeks with BM-DC-based vaccine. BM-DC/TAg cells maturing in the presence of IL-12 were excluded from the tested groups because the BM-DC/IL-12 cells were the source of IL- 12 .

Only the application of the BM-DC/TAg + BM-DC/IL-12 cell combination enhanced the difference in TRV ( $\triangle \mathrm{TRV}$ up to 

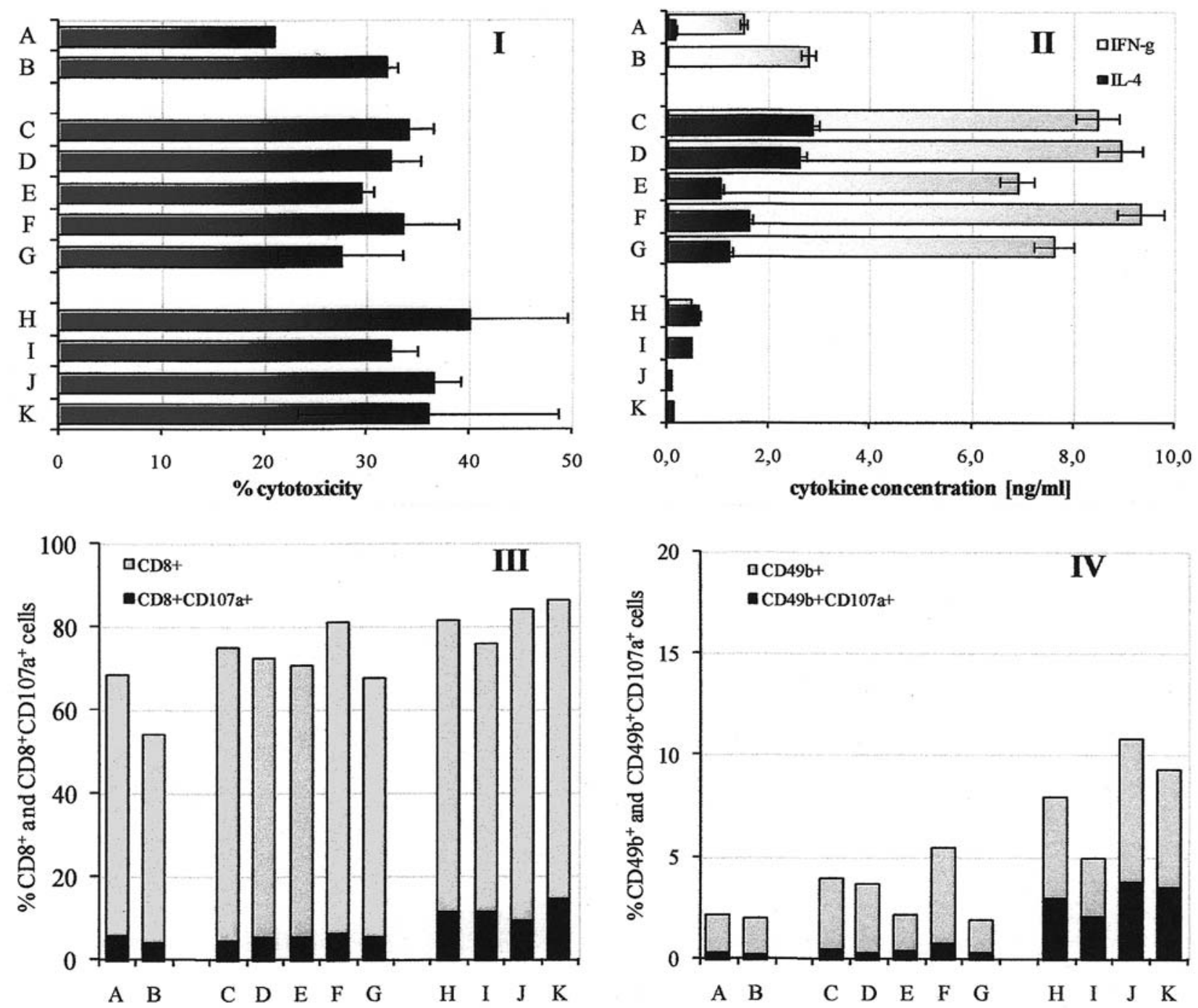

Figure 5. Immune response after vaccination with BM-DC/TAg at different stages of maturity. Spleens were dissected one week and one month after the third BM-DC injection. Then the spleen cells (splc) were restimulated with MC38/0 cells in vitro for five days. After that time the cytotoxic activity of the cells (I), their ability to produce IFN- $\gamma$ and IL-4 (II) or their ability to release cytolytic granules and expression of CD107a by CD8 $8^{+}$cells (III) and CD49+ cells (IV) were estimated. \% cytotoxicity - percentage of dead (target) MC38 cells. The concentrations of IFN- $\gamma$ and IL-4 were measured by ELISA assay in the supernatants collected from the restimulated splc after BM-DC vaccination. For the assays, splc from: healthy mice (A), untreated MC38 tumour-bearing mice (B), mice on the seventh day after the last injection of BM-DC/TAg (C), BM-DC/TAg+IL-12 (D), BM-DC/TAg+IL-12+IFN- $\gamma(\mathrm{E}), \mathrm{BM}-\mathrm{DC} / \mathrm{TAg}+\mathrm{IFN}-\gamma(\mathrm{F})$, and BM-DC/TAg+IFN- $\gamma+$ TNF- $\alpha(\mathrm{G})$ and from mice which survived up to one month after the last injection of: BM-DC/TAg (H), BM-DC/TAg+IL-12+IFN- $\gamma(\mathrm{I})$, BM-DC/TAg+IFN- $\gamma(\mathrm{J})$, and BM-DC/TAg+IFN- $\gamma+\mathrm{TNF}-\alpha(\mathrm{K})$ were used. To calculate the mean and standard deviation three mice from each presented group were tested.

11.5 days) and TGI (up to $65.5 \%$ on day 36 , Fig. 7). However, there was no statistical difference in tumour growth inhibition. The other cell vaccines had slight impact on tumour growth delay ( $\triangle \mathrm{TRV}$ ranged from 5.5 to 7 days) and TGI (32-53\%). The preliminary data indicated also that BM-DC/TAg and BM-DC/IL-12 cells applied together were able to accelerate the development of anti-tumour response more effectively than BM-DC/TAg cells used alone. Splenocytes obtained one week after the third injection (day $35)$ of BM-DC combination possessed high ability to produce IFN- $\gamma(15.5 \pm 4.5 \mathrm{ng} / \mathrm{ml})$. Moreover, high percentages of cytotoxic cells CD8 ${ }^{+} \mathrm{CD} 107 \mathrm{a}^{+}(7.5 \pm 3.4 \%)$ and $\mathrm{CD} 49 \mathrm{~b}^{+}$ $\mathrm{CD} 107 \mathrm{a}^{+}(3.8 \pm 2.5 \%)$ were detected (data not shown). In comparison with the combined BM-DC-based vaccine, the
BM-DC/TAg-based vaccine affect formation of a very low percentage of cytotoxic cells.

\section{Discussion}

Myeloid DCs, because of their ability to induce Th1-type response and $\mathrm{CD} 8^{+}$cytotoxic activity, may efficiently support tumour cell elimination and have already been applied as therapeutic vaccines against cancer. However, an effective induction of anti-tumour response requires completely mature DCs expressing high levels of co-stimulatory molecules, possessing migratory activity and a high capacity to produce IL-12. Reaching the above-mentioned conditions appeared to be more difficult than was previously expected. The results 


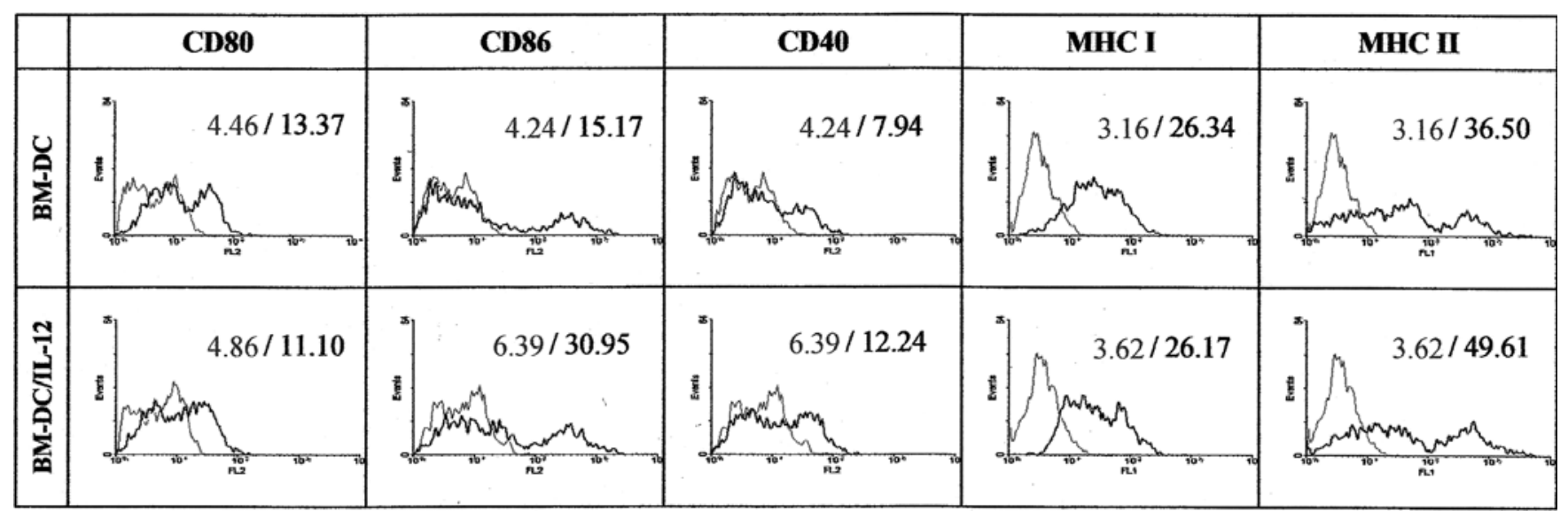

Figure 6. Phenotype characteristic of BM-DC/IL-12. The expression of CD80, CD86, CD40, MHC I, and MHC II were measured 48 h after transduction. The figure presents the results from one of four experiments. The numbers correspond to the MFI (mean florescence intensity) for the isotype control (gray) vs. the examined surface antigen (black)

obtained until now demonstrate that DCs loaded with tumour antigen do not acquire a completely mature stage, which results in their low ability to induce a tumour-specific response. However, DCs matured in the presence of inflammatory cytokines such as IL-1ß, TNF- $\alpha$ and IL-6 reached a higher stage of maturity and proved to be more efficient activators of immune response in vitro (13). It was also reported that very long in vitro exposure to maturation stimuli leads to the development of DCs which have already exhausted their IL-12 production capacity. Consequently, the $\mathrm{T}$ cell-stimulatory properties of maturing DCs cannot be further enhanced by subsequent interactions with activated, antigen-specific $\mathrm{T}$ cells $(23,24)$. Thus, all additional stimulators of DC maturation should be used carefully.

There are studies demonstrating that DCs loaded with tumour antigen and maturing in the presence of inflammatory cytokines are efficient activators of anti-tumour immune response in vitro $(12,23)$. However, there is still not enough evidence that DC-based vaccine prepared in this way evokes similar strong effect as observed in vitro. Therefore, we investigated the influence of BM-DCs loaded with TAg and then differentiated in the presence of the inflammatory cytokines (IFN- $\gamma$, TNF- $\alpha$, IL-12) or their combinations (IFN- $\gamma$ with TNF- $\alpha$ or IL-12) on host anti-tumour immunity.

The results of the presented study confirmed that TAgloaded BM-DCs additionally stimulated with cytokines (BM-DC/TAg+/-cytokines) showed enhanced expression of co-stimulatory molecules and $\mathrm{MHC}$ antigens compared with BM-DC/TAg cells, whereas BM-DC/TAg cells alone were not able to reach complete maturity and the expression levels of the surface molecules did not increase compared with immature control BM-DCs. A similar effect was obtained by Vegh and Mazumder (12) in experiments performed in vitro on human DCs generated from peripheral blood mononuclear cells. They reported that DCs first loaded with tumour lysate and further treated with a combination of TNF- $\alpha$ and IFN- $\gamma$ showed complete maturity.

Thus, BM-DC/TAg cells maturing in the presence of the inflammatory cytokines are more efficient activators of the
Th1-type immune response in vitro than partially differentiated BM-DC/TAg cells. Naïve spleen cells co-cultivation for five days with BM-DC/TAg+cytokines cells resulted in a high intracytoplasmic level of IFN- $\gamma$. However, the IFN- $\gamma$ level in supernatants collected after 5 day co-cultivation was significantly higher only when the spleen cells were activated by BM-DC/TAg+IL-12 (101 ng/ml) or BM-DC/TAg+IFN- $\gamma$ $+\mathrm{IL}-12(108 \mathrm{ng} / \mathrm{ml})$ cells. The other tested groups of BM-DC/ TAg activated splenocytes to moderate production of IFN- $\gamma$ (up to $30 \mathrm{ng} / \mathrm{ml}$ ). These huge discrepancies may be explained by the fact that BM-DCs stimulated additionally with IL-12 most likely forced splenocytes to earlier IFN- $\gamma$ production than other BM-DCs and therefore cumulative concentration of the cytokine in 5-day supernatant was higher.

We also assessed the effect of partially differentiated BM-DC/TAg cells as well as those differentiated in the presence of inflammatory cytokines on tumour growth inhibition. The injection of the BM-DC-based vaccines resulted only in insignificant tumour growth delay $(\triangle T R V$; up to 4 days) and low tumour growth inhibition (TGI: 29$44 \%)$. Unexpectedly, the highest $\Delta \mathrm{TRV}$ and TGI was noted when mice were injected with partially differentiated BMDC/TAg cells (Fig. 4). To assess the impact of BM-DC/TAg cells on host immune-cell activity, seven days and one month after the third injection of BM-DC/TAg cells, the animals were sacrificed and their spleens dissected. The primed in vivo splenocytes were restimulated in co-culture with MC38 cells for five days and then their cytotoxic activity as well as their ability to produce IFN- $\gamma$ and IL- 4 were analyzed. Spleen cells obtained seven days after BM-DC injection produced a high amount of IFN- $\gamma$ compared with healthy or untreated tumour-bearing control mice cells. The IL-4 production was also observed, but the IFN- $\gamma$ concentration in the culture supernatants were significantly higher. Thus, we can say that the $\mathrm{T}$ cell profile of vaccinated mice was considerably shifted towards the Th1 type. Another situation was observed one month after the third BM-DC injection. Restimulated splenocytes showed increased cytolytic activity, but they did not produce IFN $-\gamma$. Moreover, enhanced numbers of 


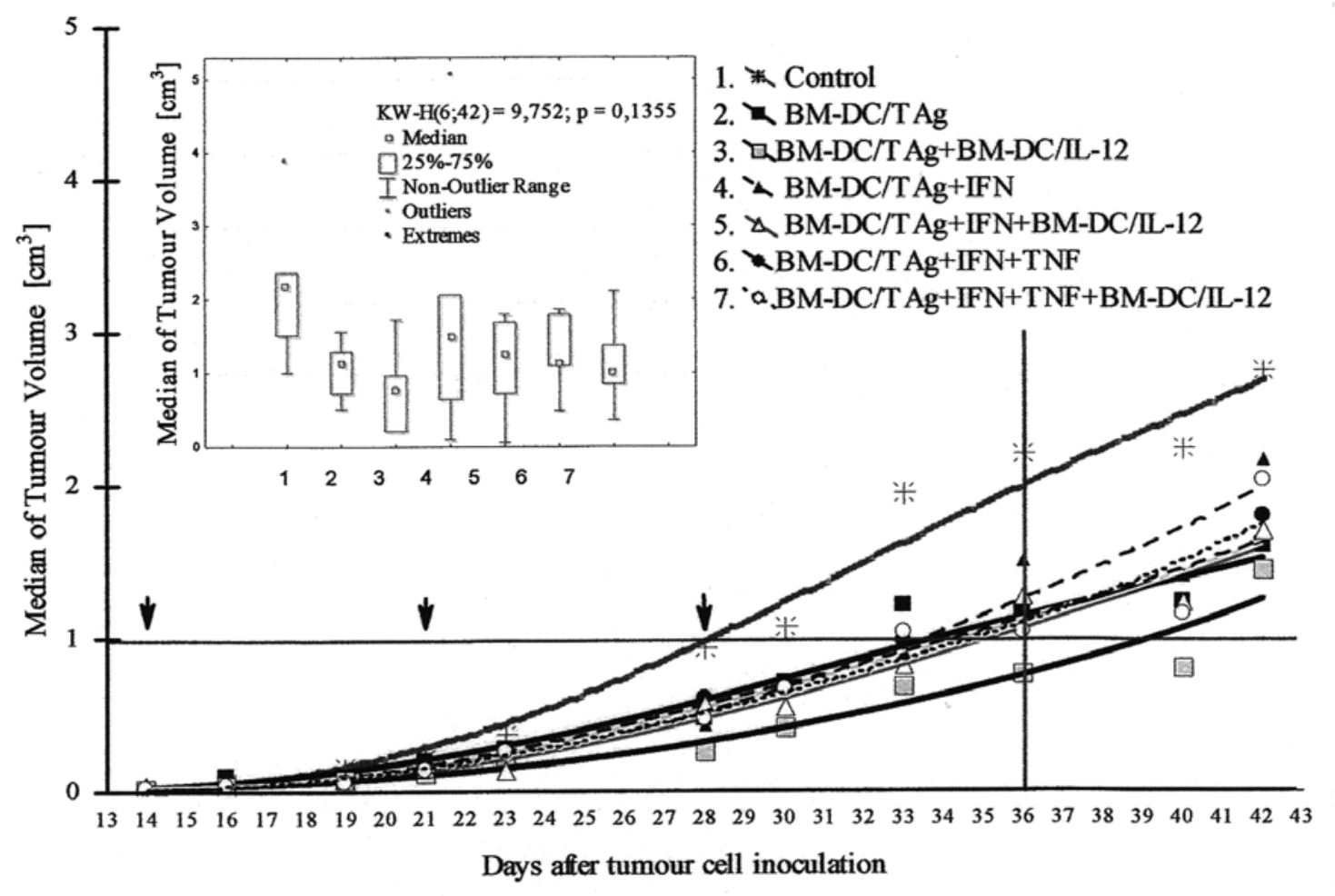

\begin{tabular}{l|c|c|c}
\hline \multicolumn{1}{c|}{ Groups } & $\begin{array}{c}\text { TRV } \\
\text { (days) }\end{array}$ & $\begin{array}{c}\Delta \text { TRV } \\
\text { (days) }\end{array}$ & $\begin{array}{c}\text { TGI } \\
{[\%]}\end{array}$ \\
\hline 1. Control & 28 & - & - \\
2. BM-DC/TAg & 34 & 6 & 47.5 \\
3. BM-DC/TAg + BM-DC/IL-12 & 39.5 & 11.5 & 65.5 \\
4. BM-DC/TAg+IFN $\gamma$ & 33.5 & 5.5 & 32 \\
5. BM-DC/TAg+IFN $\gamma+\mathrm{BM}-\mathrm{DC} / \mathrm{IL}-12$ & 35 & 7 & 43 \\
6. BM-DC/TAg+IFN $\gamma+\mathrm{TNF} \alpha$ & 34.5 & 6.5 & 47.5 \\
7. BM-DC/TAg+IFN $\gamma+\mathrm{TNF} \alpha$ & 35 & 7 & 53 \\
+BM-DC/IL-12 & & & \\
\hline
\end{tabular}

Figure 7. MC38 colon tumour growth in C57BL/6 mice treated with multiple peritumoural injections of TAg-loaded BM-DCs at different stages of maturation together with BM-DC/IL-12 cells. Mice bearing advanced s.c. growing MC38 tumours (6 per group) were injected p.t. ( $\sim 1 \times 10^{6}$ cells per injection) on three consecutive weeks (arrows in the graph). TRV, time in days required for tumour to reach a volume of $1 \mathrm{~cm}^{3} ; \Delta T R V$, difference between the TRVs of treated mice and control mice; TGI, tumour growth inhibition calculated on the $36^{\text {th }}$ day after tumour inoculation (one week after the third BM-DC injection).

cytotoxic $\mathrm{T}\left(\mathrm{CD} 8^{+}\right)$and $\mathrm{NK}\left(\mathrm{CD} 49 \mathrm{~b}^{+}\right)$cells expressing surface CD107a molecule were present. The results demonstrated that BM-DC-based vaccination, both with partially and complete mature cells, may activate mechanisms of host cell cytotoxicity, which play a crucial role in anti-tumour response.

Our experiments showed that evaluation of the BM-DC maturity stage and their ability to in vitro activation of a $\mathrm{T}$ cell response do not provide enough information on the in vivo functionality of the BM-DC-based vaccines. Partially differentiated BM-DC/TAg cells with moderate functional potential in vitro proved to be more effective activators of immune response in vivo than BM-DC/TAg cells matured in the presence of inflammatory cytokines. Findings by Woo and co-workers (25) also suggest that phenotypic and functional maturation of DCs via antigenic uptake in vitro is not necessary to successfully promote anti-tumour immunity in vivo. It is well known that the DC maturation process occurring in vivo after pathogen recognition and uptake is further modulated by concomitantly secreted pro-inflammatory cytokines. During this process, phenotypic maturation is continued, the expression of co-stimulatory and antigenpresentation molecules gradually increases, and DCs gain the ability to migrate and interact with $\mathrm{T}$ cells. Thus, it is possible that partially differentiated BM-DC/TAg cells preserve the ability to respond to additional maturation signals derived from 
hosts, while BM-DC/TAg cells, having achieved complete maturity in vitro by additional treatment with inflammatory cytokines, lose it.

The site of DC injection as well as the type and stage of tumour disease also seem to be of great importance. Experiments previously performed in our laboratory demonstrated that partially differentiated DCs stimulated with MC38 tumour cell lysate, injected p.t. into MC38 tumour-bearing mice, showed considerably reduced migration ability (26). In contrast, DCs injected s.c. into healthy mice rapidly infiltrated the lymph nodes (27). The effect of slower efflux of peritumourally injected DCs to the draining lymph nodes could be caused by immunosuppressive factors such as IL-10, TGF- 3 , VEGF, or PGE2, often produced by growing tumours, which could have an additional negative impact on the migratory efficacy of DCs (28). To change a hostile tumour environment and trigger a more efficient anti-tumour response, we decided to apply DCs transduced with IL-12 genes as an adjuvant in DC/TAg-based tumour immunotherapy. Our previous experiments showed that the dendritic cell line JAWS II transduced with IL-12 genes applied in combination with partially differentiated JAWS II/TAg (22) or BM-DC/IL-12 with BM-DC/TAg significantly increase the effectiveness of TAg-loaded DCs in evoking of antitumour response. But the effect was observed only when partially differentiated BM-DC/TAg cells were applied (Fig. 7). The results suggest that IL-12 produced by transduced DCs in the vicinity of the tumour changed the hostile tumour environment as well as enhanced the maturity stage of the injected p.t. DC/TAg cells.

Summarizing, our results provide strong evidence that tumour antigen-loaded BM-DCs maturing in the presence of inflammatory cytokines can be more effective activators of immune response in vitro but not in vivo. It should be taken into consideration that the DC maturation process occurring in vivo depends not only on antigenic stimulation, but also on the sequence of other events such as the production of proinflammatory cytokines. It seems that completely matured DCs injected as an anti-tumour vaccine are not capable of reacting to all the additional signals taking place in vivo. These cells may thus contribute to the development of an impaired immune response. On the other hand, it seems probable that partially differentiated DCs preserve their capacity for further differentiation and sensitivity to factors secreted by the host after DC injection. However, the host response to peri-tumourally injected DC/TAg cells may be too weak to trigger the next step in maturation. In this case, the vaccination with genetically modified DCs, producing inflammatory cytokines such as IL-12, together with DC/TAg cells, seems to be advisable.

\section{Acknowledgements}

This study was supported by the Ministry of Science and Higher Education (grants PBZ-KBN-091/PO5/2003 and 2798/PO1/2007/32).

\section{References}

1. Abbas AK and Sharpe AH: Dendritic cells giveth and taketh away. Nat Immunol 6: 227-228, 2005.
2. Byrne $\mathrm{S}$ and Halliday G: Dendritic cells: making progress with tumour regression? Immunol Cell Biol 80: 520-530, 2002.

3. Yoshida S, Morii K, Watanabe M, Saito T, Yamamoto K and Tanaka R: The generation of anti-tumoural cells using dentritic cells from the peripheral blood of patients with malignant brain tumours. Cancer Immunol Immunother 50: 321-327, 2001.

4. Iwashita Y, Tahara K, Goto S, et al: A phase I study of autologous dendritic cell-based immunotherapy for patients with unresectable primary liver cancer. Cancer Immunol Immunother 52: 155-161, 2003.

5. Kondo H, Hazama S, Kawaoka T, et al: Adoptive immunotherapy for pancreatic cancer using MUC1 peptide-pulsed dendritic cells and activated T lymphocytes. Anticancer Res 28: 379-387, 2008.

6. Guardino AE, Rajapaksa R, Ong KH, Sheehan K and Levy R: Production of myeloid dendritic cells (DC) pulsed with tumourspecific idiotype protein for vaccination of patients with multiple myeloma. Cytotherapy 8: 277-289, 2006.

7. Kobayashi T, Yamanaka R, Homma J, et al: Tumour mRNAloaded dendritic cells elicit tumour-specific CD8(+) cytotoxic T cells in patients with malignant glioma. Cancer Immunol Immunother 52: 632-637, 2003.

8. Manickan E, Kanangat S, Rouse RJ, Yu Z and Rouse BT: Enhancement of immune response to naked DNA vaccine by immunization with transfected dendritic cells. J Leukocyte Biol 61: 125-132, 1997.

9. Machy P, Serre K, Baillet M and Leserman L: Induction of MHC class I presentation of exogenous antigen by dendritic cells is controlled by $\mathrm{CD}^{+} \mathrm{T}$ cells engaging class II molecules in cholesterol-rich domains. J Immunol 168: 1172-1180, 2002.

10. Nouri-Shirazi M, Banchereau J, Bell D, et al: Dendritic cells capture killed tumour cells and present their antigens to elicit tumour-specific immune responses. J Immunol 165: 3797-3803, 2000.

11. Chen Z, Moyana T, Saxena A, Warrington R, Jia Z and Xiang J: Efficient antitumour immunity derived from maturation of dendritic cells that had phagocytosed apoptotic/necrotic tumour cells. Int J Cancer 93: 539-548, 2001.

12. Vegh Z and Mazumder A: Generation of tumour cell lysateloaded dendritic cells preprogrammed for IL-12 production and augmented $\mathrm{T}$ cell response. Cancer Immunol Immunother 52: 67-79, 2003.

13. Kaka AS, Foster AE, Weiss HL, Rooney CM and Leen AM: Using dendritic cell maturation and IL-12 producing capacity as markers of function: a cautionary tale. J Immunother 31: 359-369, 2008.

14. Mellman I and Steinman RM: Dendritic cells: specialized and regulated antigen processing machines. Cell 106: 255-258, 2001.

15. Menges M, Rößner S, Voigtländer C, et al: Repetitive injections of dendritic cells matured with tumour necrosis factor $\alpha$ induce antigen-specific protection of mice from autoimmunity. J Exp Med 195: 15-21, 2002

16. Kelleher P and Knight SC: IL-12 increases CD80 expression and the stimulatory capacity of bone marrow-derived dendritic cells. Int Immunol 10: 749-755, 1998.

17. Eskelinen EL, Tanaka Y and Saftig P: At the acidic edge: emerging functions for lysosomal membrane proteins. Trends Cell Biol 13: 137-145, 2003.

18. Alter G, Malenfant JM and Altfeld M: CD107a as a functional marker for the identification of natural killer cell activity. J Immunol Methods 294: 15-22, 2004.

19. Betts MR and Koup RA: Detection of T-cell degranulation: CD107a and b. Methods Cell Biol 75: 497-512, 2004.

20. He XZ, Wang L and Zhang YY: An effective vaccine against colon cancer in mice: use of recombinant adenovirus interleukin-12 transduced dendritic cells. World J Gastroenterol 14: 532-540, 2008.

21. Pajtasz-Piasecka E, Szyda A, Rossowska J, et al: Loss of tumourigenicity of murine colon carcinoma MC38/0 cell line after transduction with a retroviral vector carrying murine IL-12 genes. Folia Biol (Praha) 50: 7-14, 2004.

22. Pajtasz-Piasecka E, Rossowska J, Szyda A, Krawczenko A and Dus D: Generation of anti-tumour response by JAWS II mouse dendritic cells transduced with murine interleukin 12 genes. Oncol Rep 17: 1249-1257, 2007.

23. Spisek R, Bougras G, Ebstein F, Masse D, Meflah K, McIlroy D and Gregoire M: Transient exposure of dendritic cells to maturation stimuli is sufficient to induce complete phenotypic maturation while preserving their capacity to respond to subsequent restimulation. Cancer Immunol Immunother 52: 445-454, 2003. 
24. Langenkamp A, Messi M, Lanzavecchia A and Sallusto F: Kinetics of dendritic cell activation: impact on priming of Th1 Th2 and nonpolarized T cells. Nat Immunol 1: 311-316, 2000.

25. Woo SC, Kim GY, Lee CM, et al: The maturation of murine bone marrow-derived dendritic cells by tumour lysate uptake in vitro is not essential for cancer immunotherapy. Cancer Biol Ther 4: 1331-1335, 2005.

26. Rossowska J, Pajtasz-Piasecka E, Szyda A, Zietara A and Dus D: Tissue localization of tumour antigen-loaded mouse dendritic cells applied as an anti-tumour vaccine and their influence on immune response. Folia Histochem Cytobiol 4: 349-355, 2007.
27. Mullins DW and Engelhard VH: Limited infiltration of exogenous dendritic cells and naive $\mathrm{T}$ cells restricts immune responses in peripheral lymph nodes. J Immunol 176: 4535-4542, 2006.

28. Mocellin S, Wang E and Marincola FM: Cytokines and immune response in the tumour microenvironment. J Immunother 24: 392-407, 2001. 